\title{
Interoperation based Business Process Management over Crossing Social Networks
}

\author{
Yuechang Liu ${ }^{1,2}$, Yong Tang ${ }^{1}$, Jican Chen ${ }^{2}$ \\ ${ }^{1}$ School of Computer Science, South China Normal University, Guangzhou, China \\ ${ }^{2}$ School of Computer Science and Engineering, Jiaying University, \\ Meizhou, China \\ ychangliu@gmail.com,ytang@scnu.edu.cn,125914581@qq.com
}

\begin{abstract}
Due to the fact that people rely on social networking tools more and more in their work, it is necessary to integrate them with industrial Business Process Management (BPM) systems. Different from the widely exploited methodology by BPM system venders that furnishes social network capability to BPM suites, this paper decouples the two parts and binds them with a middle component. Such loosely coupled approach has the advantage of multiple crossing social networks and existing BPM tools can all be adhered together, which means great flexibility and working convenience. The method also enables people to seamlessly incorporate their work with frequently used public social networking tools (e.g. Facebook, twitter, Instagram, Weibo, etc.). At the end, the paper presents a prototype implementation of this method.
\end{abstract}

Keywords: Social Networks; Business Process Management (BPM); Interoperation; Model; Design.

\section{Introduction}

Whether self-consciously or not, social media plays more and more important in people's life. It is reported that people in average spend 4 hours in updating his/her social networks and 4 hours in conversations with friends in the networks based on a 40 hours' work week [3, 13]. Moreover, as a new kind of media social media plays important role in business promotion, advertising, public relation and customer relation management. Social network tools facilitate people connecting to the Internet and offer magnanimity business opportunity. However, such variety of the tools (e.g. Facebook, twitter, Weibo, blog, IM, etc.) also brings disadvantages: they further fragment people's time and impose additional burden of switching among them on people. The requirement that seamlessly integrate people's social networking and working arises.

Process and workflow management is effective way to improve work efficiency [3]. It is valuable to deploy process management tools on social networks, social Business Process Management (Social BPM) framework is proposed [2]. By using social BPM tools people define, execute and monitor business processes under the support of highly configurable on-line relations and information access control policy. It is reported that $80 \%$ of the adults use 1 to 3 social network tools, $40 \%$ use 2 or more such tools at the same time [3]. The variety of the tools fragments people's life time and imposes an additional burden of switching among the tools on them. It is highly required to study a social BPM that functions across multiple social media.

Basically there are two typical model for social media and BPM integration: one is social media biased, and the other traditional BPM biased[11]. For social media biased model, it is designed that the whole process lifecycle, including process design, enactment, initiation, execution intervention, monitoring, can all be accomplished via 
social media. While for traditional BPM biased model, networking function only serves as a communication (like discussing, sharing, reviewing) media among concerning users of the processes. Process Live1 (now named ARIS Cloud) from ARIS community and WebRatio2 from Object Management Group (OMG) are two respective representatives of above families. All the other social BPM tools reside outside of the two classes are categorized as "emergent suites" in [11].

The insights of this paper are following: first, it is not necessary for all of the BPM lifecycle operations to be conducted via the social media. For example, the design and enactment of processes are only duties of minor members of organizations. For some organizations the work processes don't frequently changed in everyday work. In such cases, it is not strong requirement for those organizations to design and enact the processes via social media channel. Second, people usually use variant different popular public social network tools in everyday life for different purposes. As instances, facebook and twitter for message communicating, instagram and flickr for photo sharing, LinkedIn for career hunting, Youtube for vedio sharing, and so on. It is more desirable to adhere these existing tools to people's work than inventing a "new" social networking tool. Third, though there are millions of BPM tools the standardization of those are highly studied and some well accepted models are proposed and adopted, which enables people to interoperate between these popular BPM tools. These insight makes us possible to develop a loosely coupled and interoperatable integration of social BPM model, which is the very subject of this paper.

The first step is the interoperational model for the social network. In this paper a honeycomb model[12] for social networks is employed. Business processes are modeled as well-known Business Process Execution Language (BPEL)[1]. Under the careful analysis of this honeycomb social network model (called Webb/Butterfield/Smith Model, WBS model for short)[12] of some current popular social networks, we described how to integrate WBS model with BPEL processes. Then, the structure of our model is formalized. To further understand the model, a run-on example is also utilized through the paper.

The paper is organized as follows: the target problem is described and analyzed in the first section; then, the formal model definitions is given in Section 2, followed by the section of architecture design. The last section is devoted to the conclusions, related and future works.

\section{Problem Description}

Consider the following scenario.

The BPM Company starts a new recruitment of the year. Difference from the past, the company decides to do the talents-hunter job on the Internet in this year. That is, the recruitment advertisement and application collection are all finished based on the social media. The manager of HR sector are responsible for the recruitment plan and requirement. All the other colleagues of HR sector are all responsible for posting the recruitment ads on social network platforms where they are registered. With all of the applications each HR receives and then are handed on to the HR manager, a screening procedure will be performed by the manager based on the applicant's resumes and the position requirements. The applications passing the first round screening will be replied with the offer of the opportunity of second-round interview. Otherwise they will be replied with a rejection letter. Then the process of the talents-hunting job is finished.

To summarize the task described in the above scenario, the process of the recruiting is managed by a core process engine. The information distribution and communication between the components are accomplished by (not only one) social network tool(s). Global illustration of such application can be depicted by Figure 1 . 


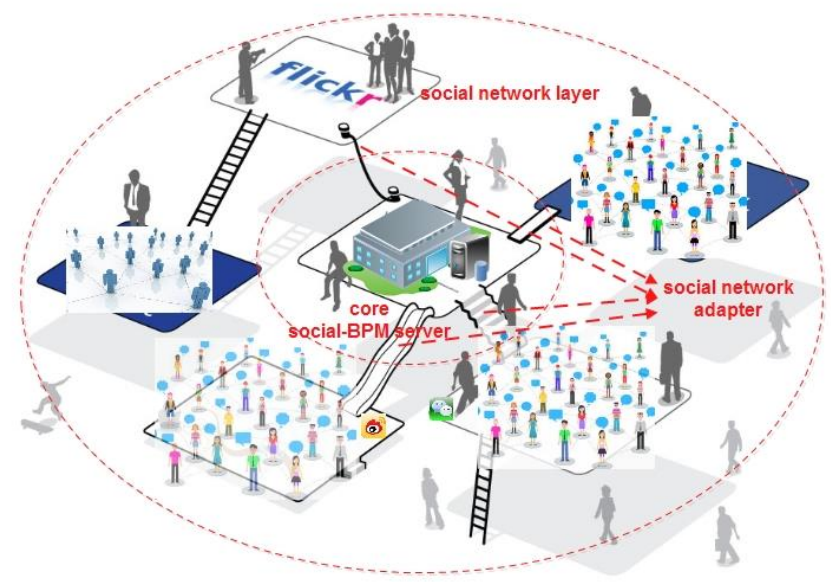

Figure 1. Social BPM Illustration

\section{Component Modeling}

\subsection{Social Network Interopeartion Model}

To accomplish the aim of this research, interoperatable model of social networks is required. In this paper, we adopt Smith's honeycomb like model (also called Webb/Butterfield/Smith model, WBS for short) [12]. In WBS model, a social network application is described as seven respects - identity, presence, relationships, reputation, groups, conversations and sharing, which can be depicted as a honeycomb (see Figure 2 below). According to WBS model, most social networks offer a combination of the functionalities in those the classes while usually focusing on a few of them. In the Figure 2, the functionalities offered and focused on by three popular social network tools are illustrated by different shades.

In Smith's theory, the classes in WBS model are further specified as Table. 1.

Considering the running example described in Section II, the recruitment process can be illustrated using traditional process chart in Figure 3.
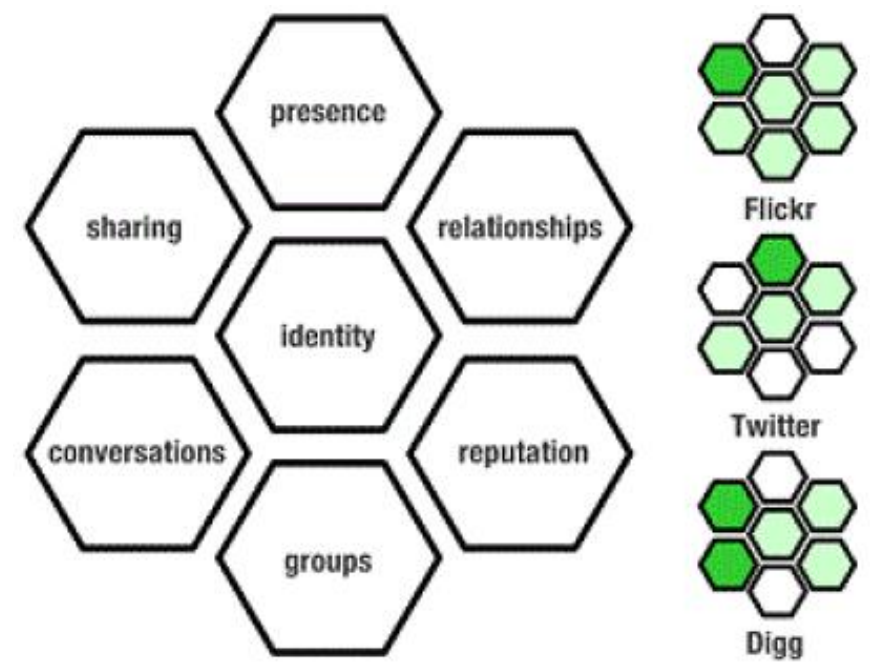

Figure 2. Webb/Butterfield/Smith Social Network Model 
Table 1. Social Network Services in WBS Model

\begin{tabular}{|c|c|c|}
\hline SNS class & Description & Attributes \\
\hline Identity & representation of a user & $\begin{array}{l}\text { Profile with personal attributes (e.g., } \\
\text { domicile), avatar (for quick indication of } \\
\text { identity), interests }\end{array}$ \\
\hline Presence & $\begin{array}{l}\text { indication of user or } \\
\text { content presence in the } \\
\text { network }\end{array}$ & $\begin{array}{l}\text { online status (e.g., available, away, busy), } \\
\text { status message, history (e.g., recently used } \\
\text { bus lines), privacy (e.g., visibility, access } \\
\text { restrictions), location (e.g., GPS coordinates, } \\
\text { bus stop name) }\end{array}$ \\
\hline Relationships & $\begin{array}{l}\text { representation of ties to } \\
\text { other people }\end{array}$ & $\begin{array}{l}\text { affiliation type (e.g., strength of tie), degree } \\
\text { of separation, directedness }\end{array}$ \\
\hline Groups & $\begin{array}{l}\text { topic-based affiliation } \\
\text { without relationship }\end{array}$ & $\begin{array}{l}\text { topic (e.g., a particular bus stop or line), } \\
\text { privacy (e.g., visibility, access restrictions) }\end{array}$ \\
\hline Content & shared information & $\begin{array}{l}\text { content annotation (none / syntactic / } \\
\text { semantic), creation dynamics (static } \\
\text { contents / dynamic participation, e.g., } \\
\text { comment functionality), forwarding (i.e., } \\
\text { sharing data of others with own contacts), } \\
\text { content types (text, link, file,...), size (e.g., } \\
\text { character or byte limit) }\end{array}$ \\
\hline Reputation & $\begin{array}{l}\text { indication of opinions } \\
\text { about contents or users }\end{array}$ & $\begin{array}{l}\text { recommendations (e.g., favorites), warnings, } \\
\text { a user's activity level }\end{array}$ \\
\hline Conversations & $\begin{array}{l}\text { communications among } \\
\text { users }\end{array}$ & $\begin{array}{l}\text { privacy (e.g., private / public messages), size } \\
\text { (e.g., character limit), dynamics (e.g., } \\
\text { comment functionality), time-dependency } \\
\text { (synchronous / asynchronous) }\end{array}$ \\
\hline $\begin{array}{l}\text { Context } \\
\text { Awareness }\end{array}$ & $\begin{array}{l}\text { adaptation to a current } \\
\text { state }\end{array}$ & $\begin{array}{l}\text { based on: location (e.g., GPS coordinates, } \\
\text { bus stop name), time, mobility, topic, } \\
\text { affiliation type (e.g., contact / group) }\end{array}$ \\
\hline $\begin{array}{l}\text { Network } \\
\text { Awareness }\end{array}$ & $\begin{array}{l}\text { indication of presence } \\
\text { and actions of other } \\
\text { users or contents }\end{array}$ & $\begin{array}{l}\text { network architecture (e.g., } \\
\text { publish/subscribe), notification (push / pull), } \\
\text { search functionality (e.g., none / user search } \\
\text { / content-based / topic-based) }\end{array}$ \\
\hline
\end{tabular}

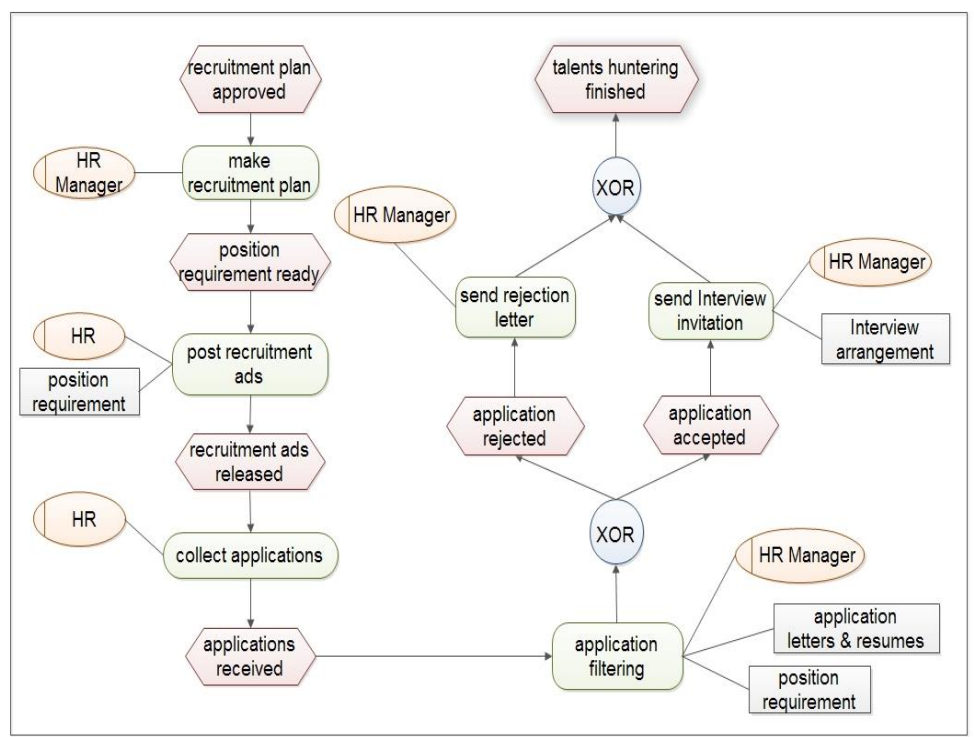

Figure 3. Traditional Process Model for the Recruitment Scenario 
In social BPM context, the process management is accomplished in social network environment, which makes it different from the traditional case. In this case, the process becomes an SOAP object that can be transmitted using HTTP.

\subsection{Conceptual Model}

Using the interoperation model defined above, the conceptual model for the crossing social media process management is illustrated as Figure 4.

\section{Cross Social Media Process Management Architecture: Design and Implementation}

The architecture is illustrated in Figure 5. As the abstraction of social networks, the Social Media Interface module separates various social network tools from the core social BPM engine. Like traditional BPM engine, the core social BPM engine serves as the core components that stores, schedules, controls and monitors the process definition and execution. The social media interface is the abstraction of the supported social networks which receives, parses and sends messages to the networks, and transforms messages to process objects (and reverse) meanwhile.

The prototype system is under implementation. Currently, only OSN interface with Sina Weibo is adopted. The implementation is based on the Weibo Open Platform (http://open.weibo.com/). Once a Weibo user logged in and add PESYS as friend, he can design a process by sending process definition text in EPML format to PESYS. Figure 7 and Figure 8 are the basic GUI of the implemented prototype system, which illustrates the dashboard and workflow management page respectively. The dashboard is mainly used for social communication function (which is synchronized with public social networks in, e.g. Weibo for now, at the back end) and information summary representation, while the process and workflow page is centered with the functions like process creation, initiation, task operation and communication. social networks in that it extends EPC with role and resource nodes to gain configurability. The model is formally defined in this paper and illustrated with a running example. 


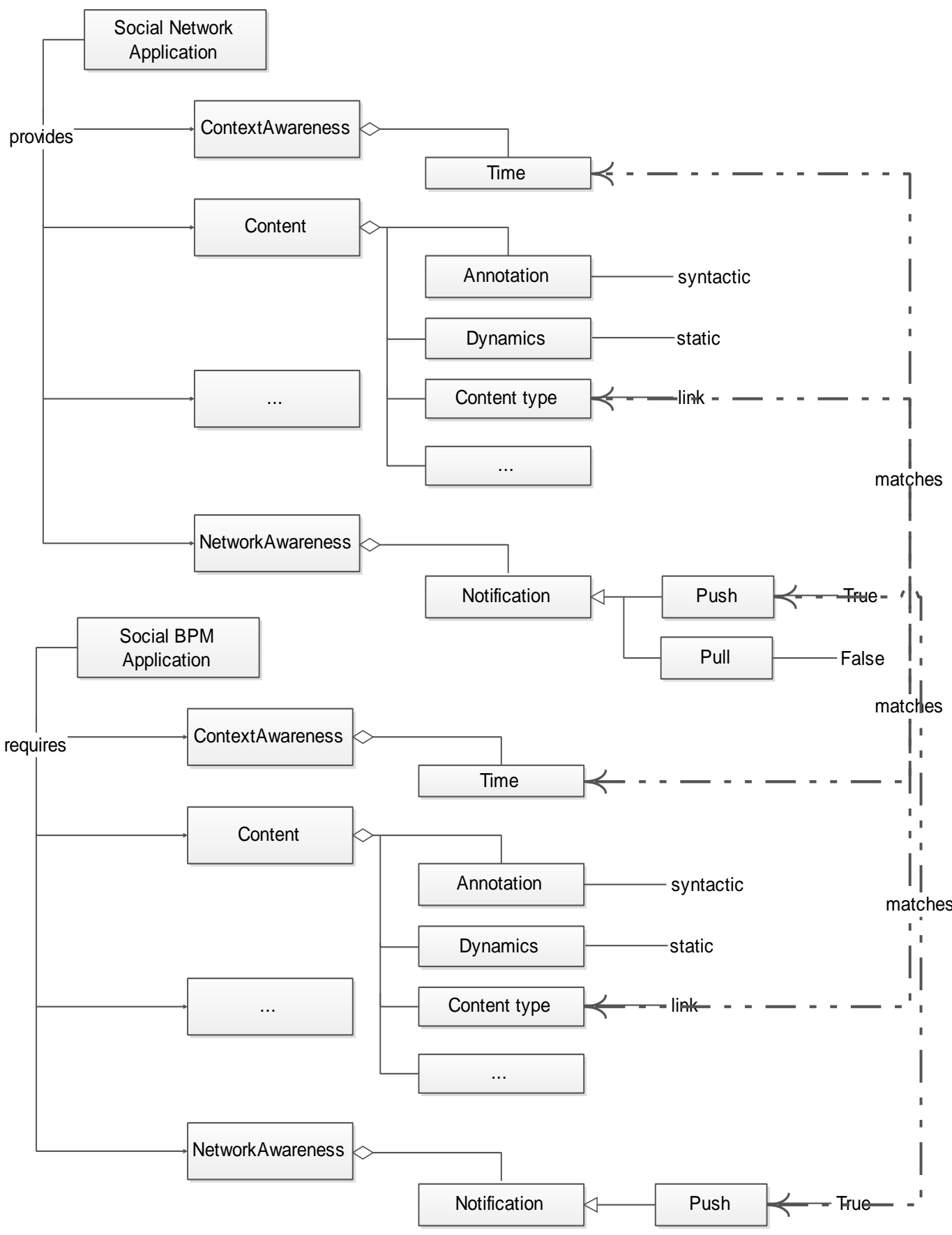

Figure 4. Conceptual Model 


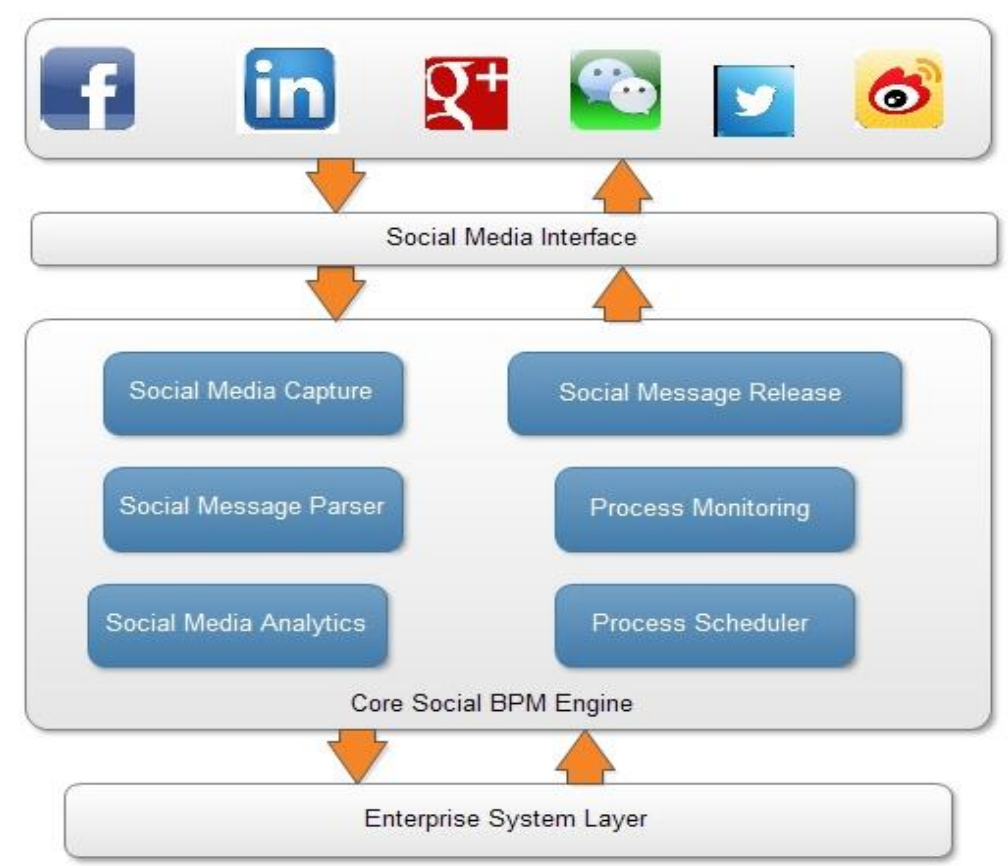

Figure 5. Crossing Social Network BPM Architecture

\section{Conclusions, Related and Future Work}

In this paper, an interoperational model based architecture that integrates traditional BPM with social networks is proposed. It adopts Smith's WBS model as interoperation model for social network description. To adapt with the social network definition, traditional process model can be organically integrated with essential social networks. The model is formally defined in this paper and illustrated with a running example.

The theory and application of social BPM has been intensively studied in recent years. It is generally recognized that social BPM is the integration of traditional BPM and social media. There are three families of solutions distinguished by the bias in the integration: tradition BPM biased, social tools biased, and emerging suites. Among of those families, social BPM suites are promising but still in their infancy. As a case of this family, IBM announced Blueworks Live [9] in mid-November 2010, but the actual social features are still under definition. Some technical community and funded research projects aim at extending traditional BPM methods towards social collaboration, like the Process Live tool from ARIS community [10]. Piero Fraternali et al proposed a model-driven framework for social BPM applications [11]. In their research, they designed a five-layer social BPM model, implemented in their WebRatio suites based on the socially extended BPMN 2.0 process modeling language and WebML (web modeling language). The research of this paper aims at designing a light-weighted social BPM mechanism, which uses existing typical social media and BPM platform. The mechanism is formalized with formal process modeling language BPEL[1] and abstract social network definition. It differs from the relevant mechanism in the literature in the process modeling language and integration tightness with social media. The model serves as the bridge between existing BPM tools and social media. In the next step, the language that is more suitable for the representation of processes in the social media will be deeply studied. Moreover, based on the formal representation language the system properties of the model will be 
further explored. In the future development, more effort will be dedicated to designing it to be an adapter middle-ware of BPM and social tools.

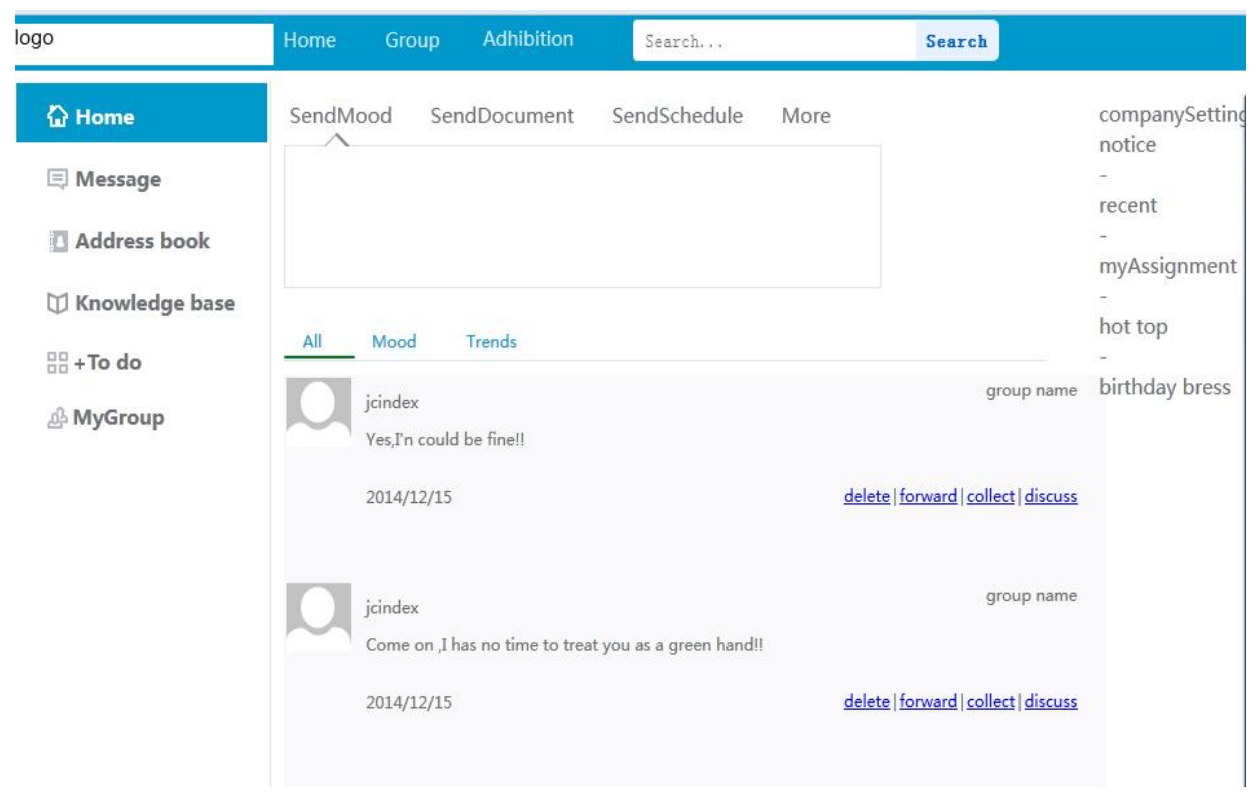

Figure 6. Dashboard Page

\begin{tabular}{|c|c|c|c|c|c|c|c|c|}
\hline \multirow{2}{*}{$\begin{array}{r}\text { Vorkflow } \\
\text { Proces }\end{array}$} & \multicolumn{3}{|c|}{ ProcessToDo I ApplicationRecord } & \multicolumn{2}{|c|}{ ApprovalRecord } & \multicolumn{2}{|c|}{ StartProcess } & Manage \\
\hline & ssToDo & & & OpenAll & AgreeAll & ProcessName & Sort & Search \\
\hline \multicolumn{9}{|l|}{ Sum:- } \\
\hline Number & Applicant & CheckProcess & StartTime & & CheckFile & \multicolumn{2}{|c|}{ Title } & Operation \\
\hline 730001 & jcindex & CheckProcess & $14-12-2$ & & RIP.pkt & \multicolumn{2}{|c|}{$x x x$ 的申请 } & ExecuteWor \\
\hline 660003 & jcindex & CheckProcess & $14-11-30$ & & & \multicolumn{2}{|c|}{ 滴答滴答滴答滴答滴答的 } & ExecuteWork \\
\hline 840010 & jcindex & $\underline{\text { CheckProcess }}$ & $14-12-7$ & & rEach.zip & \multicolumn{2}{|c|}{ jianhao的关于 $x x x$ 的申请 } & ExecuteWor \\
\hline 620003 & jcindex & $\underline{\text { CheckProcess }}$ & $14-11-30$ & & rocess.zip & \multicolumn{2}{|c|}{ 好好的1关于 $x x x$ 的申请 } & ExecuteWor \\
\hline 600007 & jcindex & CheckProcess & $14-11-30$ & & & \multicolumn{2}{|c|}{ 1好好的关于xxx的申请aa } & ExecuteWork \\
\hline 710003 & jcindex & CheckProcess & $14-12-2$ & & & \multicolumn{2}{|c|}{ 请假流程 } & ExecuteWork \\
\hline 700003 & jcindex & $\underline{\text { CheckProcess }}$ & $14-12-1$ & & & \multicolumn{2}{|c|}{ 请假流程 } & ExecuteWor \\
\hline 740003 & jcindex & $\underline{\text { CheckProcess }}$ & $14-12-2$ & & & \multicolumn{2}{|c|}{ 1好333333关于xxx的申请 $a a$} & ExecuteWork \\
\hline 600003 & jcindex & CheckProcess & $14-11-30$ & & & \multicolumn{2}{|l|}{$f$} & ExecuteWor \\
\hline 700007 & jcindex & CheckProcess & $14-12-1$ & & & \multicolumn{2}{|c|}{ 请假流程 } & ExecuteWork \\
\hline 700011 & jcindex & CheckProcess & $14-12-1$ & & & \multicolumn{2}{|c|}{ 请假流程 } & ExecuteWork \\
\hline 760003 & jcindex & CheckProcess & $14-12-4$ & \multicolumn{2}{|c|}{$\frac{\text { svn eclipse插件site- }}{\underline{1.0 .6 . z i p}}$} & \multicolumn{2}{|c|}{ 1好的请假的申请 } & ExecuteWork \\
\hline 670003 & jcindex & CheckProcess & $14-11-30$ & & & 好好的关于 & 的申请 & ExecuteWork \\
\hline
\end{tabular}

Figue 7. Process (Workflow) Management Page 


\section{Acknowledgements}

The research is supported by following projects: the National Science Foundation (No. 60970044 and No. 61272067) in part and Jiaying University Natural Science Research Project "Research and Application of Collaboration Mechanism in Social Networks".

\section{References}

[1] A. Tony, et al. "Business process execution language for web services." (2003).

[2] J. Mendling, M. Nüttgens, EPC markup language (EPML): an XML-based interchange format for eventdriven process chains (EPC), ISeB, vol. 4, no. 3, Jul. (2006), pp. 245-263.

[3] K. Shankar. "Social Process Design, Execution and Intelligence for a better Customer Experience." Infosys, white paper, (2011).

[4] M. L. Rosa, M. Dumas, A. H. M. ter Hofstede, J. Mendling and F. Gottschalk, Beyond Control-Flow: Extending Business Process Configuration to Roles and Objects, in Conceptual Modeling - ER 2008, Q. Li, S. Spaccapietra, E. Yu and A. Olivé, Springer Berlin Heidelberg, (2008), pp. 199-215.

[5] M. La Rosa, M. Dumas, A. H. M. ter Hofstede and J. Mendling, "Configurable multi-perspective business process models", Information Systems, vol. 36, no. 2, (2011), pp. 313-340.

[6] M. Smiciklas, "Social Media Workflow", Via http://www.intersectionconsulting.com/2013/social-mediaworkflow/. January 3, (2013).

[7] W. Robison, "Social Workflow - A Future Of Work Trend", Technology, January 27, (2013).

[8] D. Ferraiol, J. Cugin, D R Kuhn, "Role-based access control (RBAC): Features and motivations[C]", //Proceedings of 11th annual computer security application conference. (1995), pp. 241-48.

[9] [IBM11] IBM. IBM BlueWorks Live, 2011. https://www.blueworkslive.com, Last accessed: May (2011).

[10] Process Live http://processlive.com/

[11] M. Brambilla, P. Fraternali and C. Vaca Ruiz, "A model-driven approach to Social BPM applications", In Social BPM Handbook, BPM and Workflow Handbook series, Future Strategies, USA, (2011), pp. 95-112.

[12] K. Tietze and T. Schlegel, "On Modeling a Social Networking Service Description," (2011).

[13] J. Heidemann, M. Klier, and F. Probst, "Online social networks: A survey of a global phenomenon," Computer Networks, vol. 56, no. 18, (2012), pp. 3866-3878.

\section{Authors}
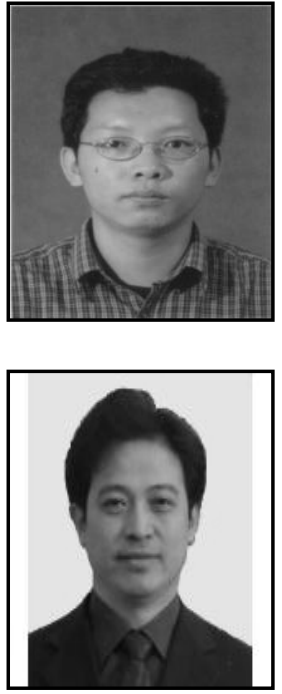

Yuechang Liu, He is a $\mathrm{Ph}$. D in computer software and theory, postdoctoral researcher in School of Computer Science, South China Normal University. His research interest include knowledge engineering, social computing and temporal reasoning.

Yong Tang, He is a Professor and Ph.D supervisor in computer science in School of Computer Science, South China Normal University. His research interests include temporal database, social computing and computer supported collaborative work. 
International Journal of Grid and Distributed Computing

Vol. 9, No. 1 (2016) 